\title{
Generalized Grey Target Decision Method for Mixed Attributes Based on Connection Number
}

\author{
Jinshan $\mathrm{Ma}^{1,2}$ and Changsheng $\mathrm{Ji}^{1}$ \\ ${ }^{1}$ School of Mines, China University of Mining and Technology, Xuzhou, Jiangsu 221116, China \\ ${ }^{2}$ School of Energy Science and Engineering, Henan Polytechnic University, Jiaozuo, Henan 454000, China
}

Correspondence should be addressed to Changsheng Ji; csji@cumt.edu.cn

Received 2 January 2014; Revised 28 February 2014; Accepted 30 March 2014; Published 27 April 2014

Academic Editor: Olivier Bahn

Copyright (C) 2014 J. Ma and C. Ji. This is an open access article distributed under the Creative Commons Attribution License, which permits unrestricted use, distribution, and reproduction in any medium, provided the original work is properly cited.

Grey target decision model for mixed attributes including real numbers, interval numbers, triangular fuzzy numbers, and trapezoidal fuzzy numbers is complex for its data processing in different ways and information distortion in handling fuzzy numbers. To solve these problems, the binary connection number proposed in set pair analysis is applied to unify different types of index values with their parameters' average values and standard deviations as determinacy-uncertainty vectors. Then the target center index vectors are determined by the modules of index vectors of all alternatives under different attributes. So the similarity of each index vector and its target center index vector called nearness degree can be calculated. Following, all the nearness degrees are normalized in linear method in order to be compared with each other. Finally, the optimal alternative can be determined by the minimum of all integrated nearness degrees. Case study demonstrated that this approach can not only unify different types of numbers, and simplify the calculation but also reduce the information distortion in operating fuzzy numbers.

\section{Introduction}

The grey target decision method has been widely used in many fields since Professor Deng established it [1-7]. However grey target decision method involving mixed attributes including real numbers, interval numbers, triangular fuzzy numbers, and trapezoidal fuzzy numbers is hard to deal with. Mixed attributes based decision making by the reported grey target decision method depends on the following steps: first, normalize different types of numbers individually; second, obtain the target center indices; third, calculate every alternative's distance to the target center; finally, make decision by alternatives' target center distances [8-11]. As far as the reported method is concerned, it is complex for different data processing ways. Besides it has deficiency in handling fuzzy numbers in two aspects: one is for distorting the information of the fuzzy numbers while normalizing them and the other is for enlarging the uncertainty between the fuzzy numbers when computing the alternatives' distances to target center. Thus an approach is required urgently to simplify the calculation, reduce the information distortion, and lower the uncertainty in dealing with fuzzy numbers.
So the binary $A+B i$ connection number proposed in set pair analysis (SPA) is employed to conduct this work $[12,13]$. The average values and standard deviations of all the index values of different types of numbers can be regarded as binary connection numbers' deterministic and uncertain terms. Furthermore these binary connection numbers can also be thought as the determinacy-uncertainty vectors. Based on the special vectors and the similarity theory, the generalized grey target decision model can be constructed.

Evaluating feasible alternatives may have no criterions, which is a challenging task to the decision makers. However, grey target decision method provides the decision makers a useful tool to solve this problem. Though grey target decision method has been advanced by some scholars, the method handling mixed attributes including real numbers and fuzzy numbers needs to be further studied. The proposed approach unifies the calculation ways of different types of numbers with binary connection number, does not normalize the raw data of all alternatives, and calculates alternatives' nearness degrees instead of target center distances, as is superior in theory and in practice. So the proposed approach unifying the calculation ways, reducing the information distortion, and 


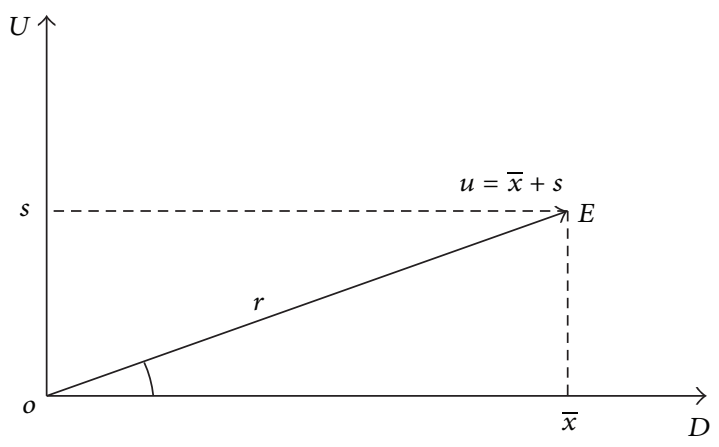

FIGURE 1: Determinacy-uncertainty space.

lowering the uncertainty in operating the fuzzy numbers may have wide use in decision making.

The remainder of this paper is organized as follows. Some basic concepts about the proposed approach are given in Section 2. Section 3 discusses the proposed approach. And the case study is presented in Section 4. Finally, Section 5 gives the conclusions.

\section{Preliminaries}

2.1. Fuzzy Number. The index values of all alternatives may be real numbers or fuzzy numbers for optimal decision making. The fuzzy number is more meaningful than that of real number, and this paper only involves interval number, triangular fuzzy number, and trapezoidal fuzzy number [14-19].

Definition 1. Let $R$ be a set of real numbers; if $\tilde{x}=\left[x^{-}, x^{+}\right]$, then $\tilde{x}$ is called interval number, where $x^{-}, x^{+} \in \mathbf{R}, x^{-}<x^{+}$.

Definition 2. Let $R$ be a set of real numbers; if $\tilde{x}=\left[x^{L}, x^{M}, x^{N}\right]$, then $\tilde{x}$ is called triangular fuzzy number, where $x^{L}, x^{M}, x^{N} \in \mathbf{R}, 0<x^{L}<x^{M}<x^{N}, x^{L}$, and $x^{N}$ are the triangular fuzzy number's lower limits and upper limits, respectively, while $x^{M}$ is the medium value.

Definition 3. Let $R$ be a set of real numbers; if $\tilde{x}=$ $\left[x^{L}, x^{M}, x^{N}, x^{U}\right]$, then $\tilde{x}$ is called trapezoidal fuzzy number, where $x^{L}, x^{M}, x^{N}, x^{U} \in \mathbf{R}, 0<x^{L}<x^{M}<x^{N}<x^{U}, x^{L}$, and $x^{U}$ are the trapezoidal fuzzy number's lower limits and upper limits, respectively, while $\left[x^{M}, x^{N}\right]$ is the medium interval.

2.2. Connection Number. The connection number is a key concept in set pair analysis founded by Zhao in 1989. It unifies the related two sets with identity, discrepancy, and contrary $[12,13]$. Now, the connection number has been used in many fields such as artificial intelligence. And the binary connection number considering the determinacy and uncertainty about two sets has also been widely used [20].

Definition 4. Let $R$ be a set of real numbers; then $A+$ $B i$ is called binary connection number, where $A, B \in$ $R, i \in[-1,1], A$ denotes the deterministic term, $B$ denotes the uncertain term, and $i$ is a variable term unifying the determinacy and the uncertainty of a set pair.

2.3. Transform Fuzzy Number into Connection Number. Zhao proposed that a fuzzy number with uncertainty can be expressed as a binary connection number. In Zhao's viewpoint, the average value and standard deviation in statistics are also the parameters of fuzzy numbers (interval number, triangular fuzzy number, or trapezoidal fuzzy number). Thus, the fuzzy number can be characterized as the binary "average value + standard deviation" connection number [14].

Suppose $\tilde{x}$ is a fuzzy number (interval number, triangular fuzzy number, or trapezoidal fuzzy number); if its parameters can be seen as $n(n \geq 2)$ observed values, then the fuzzy number's average value and standard deviation are as follows:

$$
\begin{gathered}
\bar{x}=\frac{1}{n} \sum_{t=1}^{n} x_{t}, \\
s=\sqrt{\frac{1}{(n-1)} \sum_{t=1}^{n}\left(x_{t}-\bar{x}\right)^{2} .}
\end{gathered}
$$

According to the SPA, the average value $\bar{x}$ and the standard deviation $s$ reflect 2 parameters of $n$ observed values for the same object $x$, so they can be regarded as the related two sets and form the set pair $H=(\bar{x}, s)$, where $\bar{x}$ and $s$ can also be thought as the relative deterministic (concentration) measure and the relative uncertain (dispersion) measure of $n$ observed values about $x$. So $H=(\bar{x}, s)$ is a determinacyuncertainty set pair and the relationship of $\bar{x}$ and $s$ denoted by the binary connection number $A+B i$.

Definition 5. Let $\bar{x}$ and $s$ be the average value and the standard deviation of $n(n \geq 2)$ observed values of the same object $x$, respectively; then

$$
u(\bar{x}, s)=A+B i=\bar{x}+s i \quad(i \in[-1,1])
$$

is called the average value-standard deviation connection number of $n$ observed value of the object $x$ or connection number for short.

Definition 6. The mutual interaction of $\bar{x}$ and $s$ of the average value-standard deviation connection number $u(\bar{x}, s)$ can be mapped to the binary determinacy-uncertainty space $(D-U$ space) based on SPA. If $u(\bar{x}, s)=\bar{x}+s i$ represents the vector in the $D-U$ space, then the " $i$ " only denotes the signal of the uncertain term without representing the variable value.

Figure 1 is a $D-U$ space. The $U$ axis represents the relative uncertain measure, while the $D$ axis is the relative deterministic measure. Seen from Figure 1, $\bar{x}$ and $s$ interact with each other, and the space reflection is the vector $\overline{O E}$ from $O$ to $E$, and the degree of interaction means the module of the vector $\overline{O E}$ denoted by $r$ [14].

2.4. Similarity Measure between Vectors. Measure between vectors includes distance method [21] and similarity method [22], and this paper only refers to the latter. 
Definition 7. Let $X=\left(x_{1}, x_{2}, \ldots, x_{n}\right)$ be a vector; then

$$
|X|=\sqrt{\sum_{t=1}^{n} x_{t}^{2}}
$$

is called the module of $X$.

Definition 8. Let $X=\left(x_{1}, x_{2}, \ldots, x_{n}\right)$ and $Y=\left(y_{1}, y_{2}, \ldots, y_{n}\right)$ be two vectors; then

$$
\operatorname{sim}(X, Y)=\cos (X, Y)=\frac{\sum_{t=1}^{n} x_{t} y_{t}}{\sqrt{\sum_{t=1}^{n} x_{t}^{2} \sum_{t=1}^{n} y_{t}^{2}}}
$$

is the cosine of $X$ and $Y$. The cosine value reflects the similarity of $X$ and $Y$, and the bigger of the cosine value is the more similarity of them.

Definition 9. Let $X=\left(x_{1}, x_{2}, \ldots, x_{n}\right)$ and $Y=\left(y_{1}, y_{2}, \ldots, y_{n}\right)$ be two vectors; then

$$
\operatorname{Prj}_{Y}(X)=\frac{\sum_{t=1}^{n} x_{t} y_{t}}{\sqrt{\sum_{t=1}^{n} x_{t}^{2} \sum_{t=1}^{n} y_{t}^{2}}} \sqrt{\sum_{t=1}^{n} x_{t}^{2}}=\frac{\sum_{t=1}^{n} x_{t} y_{t}}{\sqrt{\sum_{t=1}^{n} y_{t}^{2}}}
$$

is the projection of $X$ on $Y$. Obviously, the bigger value of $\operatorname{Prj}_{Y}(X)$ is the more similarity between $X$ and $Y$ [23].

\subsection{Grey Target Decision Method and Its Generalized Method.} Seeking for the satisfied alternative is the essence of grey target decision method. Actually, it is impossible to obtain the absolute optimal alternative, so the satisfied one is accepted. The definitions of grey target decision method and its generalized method are as follows.

Definition 10. When evaluating multiattribute alternatives without standard model, a grey target is set and the target center is derived from it in order to calculate every alternative's distance to target center for decision making, which is called grey target decision method.

Definition 11. Based on the grey target decision theory, however, the method of procedure and handling technique that is different from the classical one is called generalized grey target decision method. Compared with the traditional model, the generalized method has two differences: needless to normalize the index values and difference of target center distance calculation.

The proposed generalized approach for handling mixed attributes has its advantages over the previous reported method. First, it uses the uniform way to handle different types of numbers, which is easy to operate for decision makers. Second, it will reduce the information distortion in normalizing the fuzzy numbers and lower the uncertainty in calculating the target center distances, which may improve the accuracy of decision making. Third, the proposed approach which is a generalized method can be further improved and applied in a wide range fields. The generalized grey target decision method based on connection number will be presented next. However, the interested readers who want to learn the previous reported method can see the literature $[8-11,24]$.

\section{Generalized Grey Target Decision Method for Mixed Attributes}

In this section, the basic work for constructing the generalized grey target decision model is first conducted and then the algorithm of the approach is concluded.

3.1. Transform Index Values into Connection Numbers. Let $C=\left\{C_{1}, C_{2}, \ldots, C_{n}\right\}$ be an alternative set and let $A=$ $\left\{A_{1}, A_{2}, \ldots, A_{m}\right\}$ be an attribute set; then the index of alternative $C_{s}$ under attribute $A_{t}$ is $v_{s t}(s=1,2, \ldots, n$; $t=1,2, \ldots, m)$. Here the mixed attributes may be expressed as real numbers and fuzzy numbers. And the benefit type index set and cost type index set are denoted by $J^{+}$and $J^{-}$, respectively.

Using the equations from (1) to (2), the different types of index values can be converted into binary $A+B i$ connection numbers thought as the vectors in the $D-U$ space. Note that the converted binary connection number for real number is the form $A+0 i$, which means the deterministic term is the real number itself and the uncertain term is $0 i$. The transformed index vector can be expressed as

$$
U_{s t}=A_{s t}+B_{s t} i \quad(s=1,2, \ldots, n ; t=1,2, \ldots, m),
$$

where $A_{s t}$ and $B_{s t}$ denote the average value and standard deviation of $v_{s t}$, respectively. And the module of $U_{s t}$ can be calculated using the following equation:

$$
\left|U_{s t}\right|=\sqrt{A_{s t}^{2}+B_{s t}^{2}} \quad(s=1,2, \ldots, n ; t=1,2, \ldots, m) .
$$

3.2. Determine the Target Center Index Vectors. Having got the binary connection numbers converted from all index values using (6), the target center index vectors can be determined according to the following equation:

$$
\begin{gathered}
C_{t}^{0}=\left\{\begin{array}{l}
\left\{U_{s t} \mid \max \left\{\left|U_{s t}\right|\right\}, U_{s t} \in J^{+}\right\} \\
\left\{U_{s t} \mid \min \left\{\left|U_{s t}\right|\right\}, U_{s t} \in J^{-}\right\},
\end{array}\right. \\
s=1,2, \ldots, n, \quad t=1,2, \ldots, m .
\end{gathered}
$$

However, if the index vectors corresponding to $\max \left\{\left|U_{s t}\right|\right\}$ or $\min \left\{\left|U_{s t}\right|\right\}$ are not unique, then choose the vector with the minimum uncertain term $B_{s t} i$ of $U_{s t}$ such that $\min \left\{\left|B_{s t}\right|\right\}$ is target center index vector. The equation is as follows:

$$
\begin{array}{r}
C_{t}^{0}=\left\{\begin{array}{r}
\left\{U_{s t} \mid \max \left\{\left|U_{s t}\right|\right\}, \min \left\{\left|B_{s t}\right|\right\}, U_{s t} \in J^{+}\right\} \\
\left\{U_{s t} \mid \min \left\{\left|U_{s t}\right|\right\}, \min \left\{\left|B_{s t}\right|\right\}, U_{s t} \in J^{-}\right\},
\end{array}\right. \\
s=1,2, \ldots, n, \quad t=1,2, \ldots, m .
\end{array}
$$

Equations (8) and (9) tell that the target center index vector under attribute $t$ is that the index vector with the 


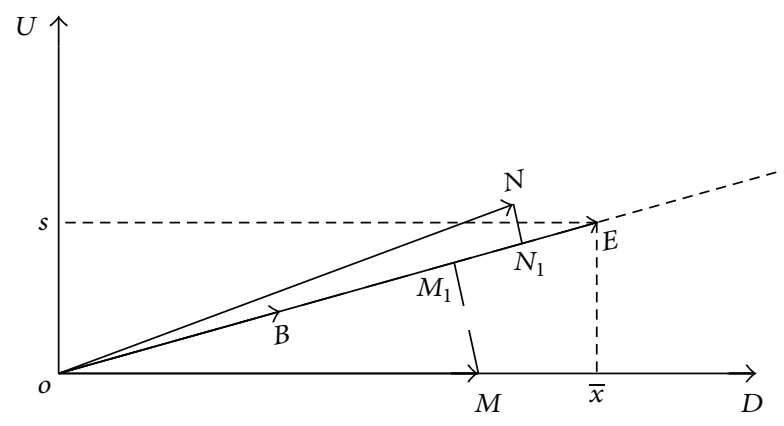

Figure 2: The relationship of different types of index vectors and the baseline index vector.

maximum module for benefit type indices and that with the minimum module for cost type indices. However, if the vectors to the maximum or the minimum module are not unique, the target center index vector is the one with the minimum uncertain term of these index vectors.

\subsection{Nearness Degrees Calculation}

(1) The Deficiency of Using Distance to Measure the Fuzzy Numbers. The decision making may not be as accurate as expected with the fuzzy numbers operated by distance which actually enlarges the uncertainty of them. Two aspects lead to the uncertainty: one is the information distortion when normalizing the fuzzy numbers, and the other is the operation of calculating the distances between the fuzzy numbers. However, the measure of the fuzzy numbers by vector similarity is superior to that by distance in theory for it is not normalizing the fuzzy numbers and also considering the fuzzy number's determinacy-uncertainty with connection number.

(2) Method Selection for the Similarity between Vectors. The cosine value reflects the similarity of the two vectors. Taking the vectors $X$ and $Y$ for an example, the bigger the value is, the more the similarity of the two vectors is. Due to the gap difference of index vectors for each attribute, the similarity between the vectors cannot always be done well only by cosine value. And the vector projection method also has its disadvantage. Figure 2 illustrates the use of cosine value and vector projection.

Figure 2 is a $D-U$ space. In the $D-U$ space, $\overline{O E}$ is a baseline vector (target center index vector), $\overline{O B}$ has the angle 0 with $\overline{O E}, \overline{O M}$ is an entirely deterministic vector, and $\overline{O N}$ has a small angle with $\overline{O E}$. Judging from the cosine value, $\overline{O B}$ is the nearest to $\overline{O E}, \overline{O N}$ is nearer to $\overline{O E}$, and $\overline{O M}$ is the furthest to $\overline{O E}$. But this relationship does not reflect the different indices near the target center index. So the vectors $\overline{O M}, \overline{O N}$, and $\overline{O B}$ should be projected to the baseline vector $\overline{O E}$ with the points $M_{1}, N_{1}$, and $B$, respectively. Thus each index near the target center index can be decided by the projection values, $\overline{O N}$ is the nearest to $\overline{O E}, \overline{O M}$ is nearer to $\overline{O E}$, and $\overline{O B}$ is the furthest to $\overline{O E}$. Note that the above conclusion using projection values to judge the indices' distances performs well only when all the projection values are smaller than the module of the baseline vector. In Figure 2, if $\overline{O B}$ is the baseline vector, then the vectors $\overline{O M}, \overline{O N}$, and $\overline{O E}$ can be projected to $\overline{O B}$ with the points $M_{1}, N_{1}$, and $E$, respectively (the points $M_{1}, N_{1}$, and $E$ can be seen as on the extended line of $\overline{O B}$ ). Then the projection value of $\overline{O E}$ on $\overline{O B}$ is the biggest among the projections, but the index distance between $\overline{O E}$ and $\overline{O B}$ is the furthest. So the effective method must be provided to conduct this work.

(3) Effective Method for Measuring the Similarity between Vectors. A new method was proposed to overcome the cosine value method and the projection value method cannot effectively solve the problem shown above. First, each index vector must be projected to the baseline vector, so the projection value can be obtained. Then, the distance between the module of baseline vector and the projection value can be calculated. And the special distance can be used to judge the distance of the two indices [25].

Definition 12. Suppose $r$ is the module of the vector $Y$ and $p_{r}$ is the projection value of the vector $X$ on the vector $Y$; then

$$
d_{n}=\left|r-p_{r}\right|
$$

is the distance of projection value $p_{r}$ and the module $r$ of the vector $Y$ is called nearness degree. When the baseline index vector and the index vector are all transformed from real numbers, $d_{n}$ is degraded to Hamming distance.

3.4. Nearness Degrees Normalization. The index values of all alternatives are not normalized; thus the nearness degrees of all index values under different attributes cannot be compared with each other directly, so the nearness degree $d_{s t}$ must be normalized. The linear normalized method is adopted to keep the information of nearness degrees (generalized target center distances). The normalized nearness degree $Z_{s t}$ can be obtained by the following equation:

$$
Z_{s t}=\frac{d_{s t}}{\sum_{s=1}^{n} d_{s t}}, \quad s=1, \ldots, n ; t=1, \ldots, m
$$

where $d_{s t}$ and $Z_{s t}$ denote the nearness degree and normalized nearness degree of $v_{s t}$, respectively and $d_{s t}$ is calculated by (10).

3.5. Weights Determination. The weights of all attributes can be determined by the deviation of the normalized nearness degrees objectively. The weight model is as follows [26].

For any two alternatives $C_{s}$ and $C_{t}$, under the attribute $j$, the deviation of nearness degree is denoted by $z_{s t j}=z_{s j}-z_{t j}$; thus all the deviations of all alternatives under attribute $j$ are as follows:

$$
z_{j}=\sum_{s=1}^{n-1} \sum_{t=s+1}^{n} z_{s j}=\sum_{s=1}^{n-1} \sum_{t=s+1}^{n}\left(z_{s j}-z_{i j}\right)
$$


TABLE 1: Index values of every alternative.

\begin{tabular}{|c|c|c|c|c|c|c|}
\hline$C_{t}$ & $A_{1}$ & $A_{2}$ & $A_{3}$ & $A_{4}$ & $A_{5}$ & $A_{6}$ \\
\hline$C_{1}$ & 2.0 & 500 & {$\left[\begin{array}{ll}55 & 56\end{array}\right]$} & {$\left[\begin{array}{ll}4.7 & 5.7\end{array}\right]$} & {$\left[\begin{array}{lll}0.4 & 0.5 & 0.6\end{array}\right]$} & {$\left[\begin{array}{lll}0.8 & 0.9 & 1.0\end{array}\right]$} \\
\hline$C_{2}$ & 2.5 & 540 & {$\left[\begin{array}{ll}30 & 40\end{array}\right]$} & {$\left[\begin{array}{ll}4.2 & 5.2\end{array}\right]$} & {$\left[\begin{array}{lll}0.2 & 0.3 & 0.4\end{array}\right]$} & {$\left[\begin{array}{lll}0.4 & 0.5 & 0.6\end{array}\right]$} \\
\hline$C_{3}$ & 1.8 & 480 & {$\left[\begin{array}{ll}50 & 60\end{array}\right]$} & {$\left[\begin{array}{ll}5 & 6\end{array}\right]$} & {$\left[\begin{array}{lll}0.6 & 0.7 & 0.8\end{array}\right]$} & {$\left[\begin{array}{lll}0.6 & 0.7 & 0.8\end{array}\right]$} \\
\hline $\mathrm{C}_{4}$ & 2.2 & 520 & {$\left[\begin{array}{ll}35 & 45\end{array}\right]$} & {$\left[\begin{array}{ll}4.5 & 5.5\end{array}\right]$} & {$\left[\begin{array}{lll}0.4 & 0.5 & 0.6\end{array}\right]$} & {$\left[\begin{array}{lll}0.4 & 0.5 & 0.6\end{array}\right]$} \\
\hline
\end{tabular}

The attribute weights can be obtained through maximizing the entire deviations, so the following model can be constructed:

$$
\begin{array}{ll}
\max & f=\sum_{j=1}^{m} z_{j} \omega_{j}^{1 / 2} \\
\text { s.t. } & \sum_{j=1}^{m} \omega_{j}=1, \quad \omega_{j} \geq 0, j=1, \ldots, m .
\end{array}
$$

To solve the nonlinear program (13) via the Lagrange function, the weights can be obtained as follows:

$$
\omega_{j}=\frac{z_{j}^{2}}{\sum_{j=1}^{m} z_{j}^{2}}, \quad j=1,2, \ldots, m,
$$

where $\omega_{j}$ is the weight of attribute $j$.

3.6. Decision Making. Every alternative's integrated nearness degree can be aggregated with every attribute's weight; the equation is as follows:

$$
w_{i}=\omega_{j} z_{i j}, \quad i=1, \ldots, n, j=1, \ldots, m .
$$

So the decision making can be obtained according to the value $w_{i}$; and the smaller value of it means the better alternative.

3.7. Algorithm of the Proposed Approach. The algorithm of the proposed approach includes the following steps.

Step 1 (measure all the feasible alternatives). The measures of feasible alternatives could be expressed as real numbers or fuzzy numbers appropriately by decision makers.

Step 2 (transform index values of all alternatives into connection numbers). The average values and standard deviations of all alternatives' indices with different types of numbers can be computed and converted into the binary $A+B i$ connection numbers using (1) and (2).

Step 3 (determine the target center index vectors). The target center index vectors can be determined by comparing with the modules of index vectors of all alternatives under different attributes using (7) and (8) or (9).

Step 4 (calculate the nearness degrees). The nearness degree of every alternative's index vector to target center index vector can be calculated using (10).
Step 5 (normalize the nearness degrees). The nearness degree of every alternative's index vector to its target center index vector can be calculated using (11).

Step 6 (determine the weights of attributes). The weights may be determined by objective method or subjective method; if the subjective method is selected, then the equations from (12) to (14) perform well.

Step 7. Aggregate the normalized nearness degrees under all attributes and the relative optimal alternative can be obtained by the minimum integrated nearness degree considering every attribute's weight use (15).

\section{Case Study}

4.1. Background. In order to exemplify the proposed generalized grey target decision method, the data are derived from the literature [8] and the results are also compared with those of the literature [8].

To evaluate the tactical missiles, six indices, namely, hit accuracy $(\mathrm{km})$, warhead payload $(\mathrm{kg})$, mobility $\left(\mathrm{km} \cdot \mathrm{h}^{-1}\right)$, price $\left(10^{6} \mathrm{~g}\right)$, reliability, and maintainability, are considered denoted by $A_{1} \sim A_{6}$. For all the data type of the attributes, $A_{1}$ and $A_{2}$ are real numbers, $A_{3}$ and $A_{4}$ are interval numbers, and $A_{5}$ and $A_{6}$ are triangular fuzzy numbers. Among these attributes $A_{1}$ and $A_{4}$ are cost type indices and the others are benefit type indices. There are four feasible alternatives denoted by $C_{1} \sim C_{4}$. The data are shown in Table 1 .

\subsection{Process to Decision Making}

(1) Calculate the Average Value and the Standard Deviation of Every Index. The average value and the standard deviation of every index can be calculated using (1), and the results are shown in Table 2. In Table $2, E_{i}$ and $s_{i}(i=1, \ldots, 4)$ represent the $i$ th alternative's average values and standard deviations of all indices under all attributes. Note that the average values and the standard deviations of real numbers are themselves and 0 , respectively.

(2) Transform Index Values into Binary Connection Numbers. Having got the average value and the standard deviation of every index shown in Table 2, every index value can be transformed into binary connection number using (2), and the results are shown in Table 3. Moreover, every binary connection number in Table 3 can be regarded as a vector in the $D-U$ space. Note that the form of the real number is expressed as the form $A+0 i(A \in R)$. 
TABLE 2: The average value and the standard deviation of every index.

\begin{tabular}{cccccccccc}
\hline$A_{t}$ & \multicolumn{2}{c}{$C_{1}$} & \multicolumn{2}{c}{$C_{2}$} & & $C_{3}$ & \multicolumn{2}{c}{$C_{4}$} \\
& $E_{1}$ & $S_{1}$ & $E_{2}$ & $S_{2}$ & $E_{3}$ & $S_{3}$ & $E_{4}$ \\
\hline$A_{1}$ & 2.0 & 0 & 2.5 & 0 & 1.8 & 0 & 0 \\
$A_{2}$ & 500 & 0 & 540 & 0 & 480 & 0 & 5.2 & 520 \\
$A_{3}$ & 55.5 & 0.707107 & 35 & 7.071068 & 55 & 7.071068 & 40 & 7.071068 \\
$A_{4}$ & 5.2 & 0.707107 & 4.7 & 0.707107 & 5.5 & 0.707107 & 5 \\
$A_{5}$ & 0.5 & 0.1 & 0.3 & 0.1 & 0.7 & 0.1 & 0.707107 \\
$A_{6}$ & 0.9 & 0.1 & 0.5 & 0.1 & 0.7 & 0.1 & 0.1 \\
\hline
\end{tabular}

TABLE 3: The binary connection numbers transformed from all index values.

\begin{tabular}{lcccc}
\hline$A_{t}$ & $C_{1}$ & $C_{2}$ & $C_{3}$ & $C_{4}$ \\
\hline$A_{1}$ & $2.0+0 i$ & $2.5+0 i$ & $1.8+0 i$ & $2.2+0 i$ \\
$A_{2}$ & $500+0 i$ & $540+0 i$ & $480+0 i$ & $520+0 i$ \\
$A_{3}$ & $55.5+0.707107 i$ & $35+7.071068 i$ & $55+7.071068 i$ & $40+7.071068 i$ \\
$A_{4}$ & $5.2+0.707107 i$ & $4.7+0.707107 i$ & $5.5+0.707107 i$ & $5+0.707107 i$ \\
$A_{5}$ & $0.5+0.1 i$ & $0.3+0.1 i$ & $0.7+0.1 i$ & $0.5+0.1 i$ \\
$A_{6}$ & $0.9+0.1 i$ & $0.5+0.1 i$ & $0.7+0.1 i$ & $0.5+0.1 i$ \\
\hline
\end{tabular}

TABLE 4: The module of every index and the target center index vector.

\begin{tabular}{lcccccc}
\hline$A_{t}$ & $r_{s 1}$ & $r_{s 2}$ & $r_{s 3}$ & $r_{s 4}$ & $r_{0}$ & $C_{0}$ \\
\hline$A_{1}$ & 2 & 2.5 & 1.8 & 2.2 & 1.8 & 540 \\
$A_{2}$ & 500 & 540 & 480 & 520 & $540+0 i$ & 54 \\
$A_{3}$ & 55.504504 & 35.707142 & 55.452683 & 40.620192 & 55.504504 & $5.5+0.707107 i$ \\
$A_{4}$ & 5.247857 & 4.752894 & 5.545268 & 5.049752 & 4.752894 & $4.7+0.707107 i$ \\
$A_{5}$ & 0.509902 & 0.316228 & 0.707107 & 0.509902 & 0.707107 & $0.7+0.1 i$ \\
$A_{6}$ & 0.905539 & 0.509902 & 0.707107 & 0.509902 & 0.905539 & $0.9+0.1 i$ \\
\hline
\end{tabular}

(3) Determine the Target Center Index Vectors. Using (6), (7), and (8) the target center index vectors can be determined by the modules of all index vectors under different attributes, and the results are shown in Table 4 . In Table $4, r_{t s}(t=$ $1,2, \ldots, 6 ; s=1, \ldots, 4)$ denotes the module of every binary connection number vector, while $r_{0}$ and $C_{0}$ are the desirable (maximum or minimum) modules of different attributes and the target center index vectors, respectively. The target center index vectors of different attributes are determined by the modules of every index vectors. The target center index vector is the one with the maximum module for benefit type attribute, while the target center index vector is the one with the minimum module for cost type attribute. For example, $A_{1}$ is a cost type attribute, so the index vector with the minimum module is $1.8+0 i$.

Finally, the target center index vector set is $C_{0}=(1.8+0 i$, $540+0 i, 55.5+0.707107 i, 4.7+0.707107 i, 0.7+0.1 i, 0.9+0.1 i)$.

(4) Calculate the Nearness Degree of Every Index Vector. Table 5 is the nearness degree of every index vector to its target center index vector calculated by the equations from (4) to (7) and (10). In Table 5, $d_{s t}(s=1, \ldots, 4 ; t=1,2, \ldots, 6)$ represents the nearness degree (generalized target center distance).

The nearness degrees of all index vectors cannot be compared with each other for different attributes, so they need to be normalized with (10). The normalized nearness degrees are shown in Table 6 . In Table $6, Z_{s t}(s=1, \ldots, 4 ; t=$ $1,2, \ldots, 6)$ represents the normalized nearness degree.

(5) Decision Making and Comparison. The entire integrated nearness degrees must be calculated for decision making. Aggregating the nearness degrees under different attributes for every alternative involves considering the attributes' weights or not.

Without considering the weights of all attributes, the entire integrated nearness degrees are calculated as $W=$ $(1.050205,2.003727,1.206973,1.739095)$, so the alternatives ranking is $C_{1}>C_{3}>C_{4}>C_{2}$.

However, if the objective weights are calculated as $\omega=$ $(0.1655,0.1534,0.3361,0.1349,0.0859,0.1241)$ by $(14)$, then the entire integrated nearness degrees $W=(0.140270$, $0.371996,0.171244,0.316391)$ can be obtained using (15), so the alternatives ranking is $C_{3}>C_{1}>C_{4}>C_{2}$. 
TABLE 5: Nearness degree of every index vector to its target center index vector.

\begin{tabular}{lcccccc}
\hline$d_{s t}$ & $A_{1}$ & $A_{2}$ & $A_{3}$ & $A_{4}$ & $A_{5}$ & $A_{6}$ \\
\hline$d_{1 t}$ & 0.2 & 40 & 0 & 0.494699 & 0.197598 & 0 \\
$d_{2 t}$ & 0.7 & 0 & 20.108669 & 0 & 0.39344 & 0.396596 \\
$d_{3 t}$ & 0 & 60 & 0.235658 & 0.791736 & 0 & 0.198604 \\
$d_{4 t}$ & 0.4 & 20 & 15.15187 & 0.29676 & 0.197598 & 0.396596 \\
\hline
\end{tabular}

TABLE 6: The normalized nearness degree of every index vector.

\begin{tabular}{ccccccc}
\hline$Z_{s t}$ & $A_{1}$ & $A_{2}$ & $A_{3}$ & $A_{4}$ & $A_{5}$ & $A_{6}$ \\
\hline$Z_{1 t}$ & 0.153846 & 0.333333 & 0 & 0.312469 & 0.250556 & 0 \\
$Z_{2 t}$ & 0.538462 & 0 & 0.566502 & 0 & 0.498887 & 0.399876 \\
$Z_{3 t}$ & 0 & 0.5 & 0.006639 & 0.500087 & 0 & 0.200247 \\
$Z_{4 t}$ & 0.307692 & 0.166667 & 0.426859 & 0.187444 & 0.250556 & 0.399876 \\
\hline
\end{tabular}

TABLE 7: The results comparison between the proposed approach and the reported method.

\begin{tabular}{ccccccccccccc}
\hline & \multicolumn{3}{c}{ No weights } & \multicolumn{4}{c}{ Objective weights } & \multicolumn{3}{c}{ Subjective weights } \\
$C_{i}$ & \multicolumn{2}{c}{$M_{1}$} & & \multicolumn{2}{c}{$M_{2}$} & \multicolumn{2}{c}{$M_{1}$} & \multicolumn{2}{c}{$M_{2}$} & $M_{1}$ & $M_{2}$ \\
& $N$ & $R$ & $D$ & $R$ & $N$ & $R$ & $D$ & $R$ & $N$ & $R$ & $D$ & $R$ \\
\hline$C_{1}$ & 1.050205 & 1 & 0.0511 & 1 & 0.140270 & 1 & 0.0888 & 2 & 0.178794 & 2 & 0.0960 & 2 \\
$C_{2}$ & 2.003727 & 4 & 0.0863 & 4 & 0.371996 & 4 & 0.2278 & 4 & 0.264120 & 4 & 0.2500 & 4 \\
$C_{3}$ & 1.206973 & 2 & 0.0661 & 2 & 0.171244 & 2 & 0.0768 & 1 & 0.150673 & 1 & 0.0783 & 1 \\
$C_{4}$ & 1.739095 & 3 & 0.0699 & 3 & 0.316391 & 3 & 0.1664 & 3 & 0.206413 & 3 & 0.1820 & 3 \\
\hline
\end{tabular}

If the weights $\omega=(0.2,0.2,0.1,0.1,0.2,0.2)$ are given by the experts, then the entire integrated nearness degrees are $W=(0.178794,0.264120,0.150673,0.206413)$, so the alternatives ranking is $C_{1}>C_{3}>C_{4}>C_{2}$.

Table 7 is the results comparison between the proposed approach and the previous method reported in literature [8]. In Table 7, $M_{1}, M_{2}, N, D$, and $R$ stand for the proposed approach, the previous reported method, integrated nearness degree, target center distance, and alternatives ranking, respectively. The objective weights determined in $M_{2}$ are $\omega=$ $(0.18,0.2,0.1,0.2,0.16,0.16)$, while the subjective weights in $M_{2}$ are $\omega=(0.2,0.2,0.1,0.1,0.2,0.2)$.

Seen from Table 7, there are differences about the results between the proposed approach and the previous reported method. Both the two methods are compared with considering the attributes with no weights, objective weights, and subjective weights. With respect to the alternatives ranking, the ranking of alternatives $C_{1}$ and $C_{3}$ is opposite, while the ranking of $C_{2}$ and $C_{4}$ remains the same only for considering the objective weights. Obviously, different objective weights make the difference. However the results of ranking with no weights and subjective weights are uniform with the two methods. Besides judging the alternatives by nearness degree is easier than by target center distance from the data results shown in Table 7 . Due to the fact that the proposed approach can reduce the information distortion, the results of it especially for considering objective weights are superior to those of the previous reported method in literature [8].

\section{Conclusions}

This paper presents a generalized great target decision method using connection number to deal with alternatives with mixed attribute values. All the index values of all alternatives can be converted into the binary connection numbers regarded as the connection number vectors in the determinacy-uncertainty space. Then the target center index vectors can be determined by the modules of the binary connection number vectors. So the nearness degrees of all index vectors can be calculated. For comparison, the nearness degrees are normalized in linear method. Thus the decision making can be made by the minimum of the integrated nearness degrees derived from aggregating the total normalized degrees of every alternative. The generalized grey target decision method for mixed attributes based on connection is superior to that of the other authors in two aspects: needless to normalize the raw data will reduce the information distortion of fuzzy numbers; considering both the determinacy and the uncertainty of the fuzzy numbers is of more accuracy.

\section{Conflict of Interests}

The authors declare that there is no conflict of interests regarding the publication of this paper. 


\section{Acknowledgments}

The authors would like to thank the support by the Key Research Project of Science and Technology of Henan Province (Grant no. 13B620033), the Natural Science Foundation of the Education Department of Henan Province (Grant no. 2011B620001), and the Henan Coal Mine Safety Production Technology Development Project (Grant no. H09-50). The authors are also grateful to the editors and the anonymous reviewers for their comments and suggestions that improved the quality of this paper.

\section{References}

[1] J. L. Deng, Grey System Theory, Huangzhong University of Science and Technology Press, Wuhan, China, 2002.

[2] Q. Zeng, H. Xie, and Q. Dai, "Grey relational analysis in clinical antibiotic selection," Journal of Grey System, vol. 20, no. 4, pp. 311-318, 2008.

[3] J. Song, Y.-G. Dang, X.-M. Li, and Z.-X. Wang, "Grey risk group decision based on the majorant operator of 'rewarding good and punishing bad"' Journal of Grey System, vol. 21, no. 4, pp. 377386, 2009.

[4] S. Chen, Z. Li, and Q. Xu, "Grey target theory based equipment condition monitoring and wear mode recognition," Wear, vol. 260, no. 4-5, pp. 438-449, 2006.

[5] J. Wu, X. Tian, Y. Tang, Y. Zhao, Y. Hu, and Z. Fang, "Application of analytic hierarchy process-grey target theory systematic model in comprehensive evaluation of water environmental quality," Water Environment Research, vol. 82, no. 7, pp. 633-641, 2010.

[6] S.-W. Chen, Z.-G. Li, and Q.-S. Xu, "Research on wear pattern recognition based on grey target theory and oil monitoring," Journal of China University of Mining \& Technology, vol. 34, no. 3, pp. 353-357, 2005.

[7] B. Liang, B. Qin, and W.-J. Sun, "Possibility assessment of coalgas outburst based on grey target model," Journal of the China Coal Society, vol. 36, no. 12, pp. 1974-1978, 2011.

[8] C. G. Shen, Y.-G. Dang, and L. L. Pei, "Hybrid multi-attribute decision model of grey target," Statistics and Decision, no. 12, pp. $17-20,2010$.

[9] Y.-G. Dang, S. F. Liu, and B. Liu, "Study on the multi-attribute decision model of grey target based on interval number," Engineering Science, no. 8, pp. 31-35, 2005.

[10] D. Luo and X. Wang, "The multi-attribute grey target decision method for attribute value within three-parameter interval grey number," Applied Mathematical Modelling, vol. 36, no. 5, pp. 1957-1963, 2012.

[11] J. Song, Y.-G. Dang, Z.-X. Wang, and X.-M. Li, “The decisionmaking model of harden grey target based on interval number with preference information on alternatives," Journal of Grey System, vol. 21, no. 3, pp. 291-300, 2009.

[12] K.-Q. Zhao and A. L. Xuan, "Set pair theory-a new theory method of non-define and its applications," Systems Engineering, vol. 14, no. 1, pp. 18-23, 1996.

[13] K. Q. Zhao, Set Pair Analysis and Its Prelimiary Application, Zhejiang Press of Science and Technology, Hangzhou, China, 2000.

[14] K. Q. Zhao, "Decision making algorithm based on set par analysis for use when facing multiple uncertain in attributes,"
CAAI Transactions on Intelligent Systems, vol. 5, no. 1, pp. 41-50, 2010.

[15] T.-P. Lo and S.-J. Guo, "Effective weighting model based on the maximum deviation with uncertain information," Expert Systems with Applications, vol. 37, no. 12, pp. 8445-8449, 2010.

[16] Z. Yue, "An extended TOPSIS for determining weights of decision makers with interval numbers," Knowledge-Based Systems, vol. 24, no. 1, pp. 146-153, 2011.

[17] L. Liu, J.-J. Chen, G.-M. Wang, and D.-Z. Lao, "Multi-attributed decision making for mining methods based on grey system and interval numbers," Journal of Central South University, vol. 20, no. 4, pp. 1029-1033, 2013.

[18] A. I. Ban and L. Coroianu, "Nearest interval, triangular and trapezoidal approximation of a fuzzy number preserving ambiguity," International Journal of Approximate Reasoning, vol. 53, no. 5, pp. 805-836, 2012.

[19] J. M. Merigó and M. Casanovas, "Decision-making with uncertain aggregation operators using the Dempster-Shafer belief structure," International Journal of Innovative Computing, Information and Control, vol. 8, no. 2, pp. 1037-1061, 2012.

[20] K. Q. Zhao, "The theoretical basis and basic algorithm of binary connection $\mathrm{A}+\mathrm{Bi}$ and its application in AI," CAAI Transactions on Intelligent Systems, vol. 3, no. 6, pp. 476-486, 2008.

[21] Z. Xu and M. Xia, "Distance and similarity measures for hesitant fuzzy sets," Information Sciences, vol. 181, no. 11, pp. 2128-2138, 2011.

[22] J. Ye, "Cosine similarity measures for intuitionistic fuzzy sets and their applications," Mathematical and Computer Modelling, vol. 53, no. 1-2, pp. 91-97, 2011.

[23] G. W. Wei, Theory and Methods of Multiple Attribute Decision Making Based on Fuzzy Information, China Economic Publishing House, Beijing, China, 2010.

[24] J. Zhu and K. W. Hipel, "Multiple stages grey target decision making method with incomplete weight based on multigranularity linguistic label," Information Sciences, no. 212, pp. 15-32, 2012.

[25] H. V. Pham, K. D. Tran, and K. Kamei, "Applications using hybrid intelligent decision support systems for selection of alternatives under uncertainty and risk," International Journal of Innovative Computing, Information and Control, vol. 10, no. 1, pp. 39-56, 2014.

[26] W. T. Xiong, H. Qi, and L. Q. Yong, "A novel model of determining objective weights based on the maximal deviation," Systems Engineering, vol. 28, no. 5, pp. 95-98, 2010. 


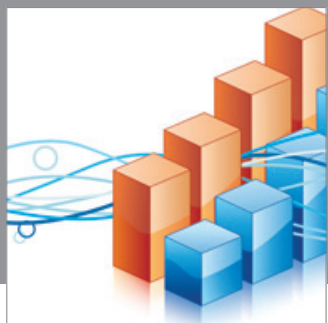

Advances in

Operations Research

mansans

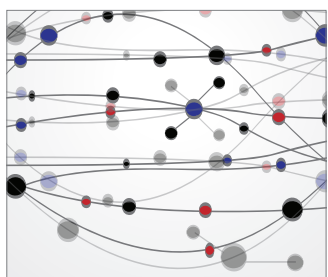

The Scientific World Journal
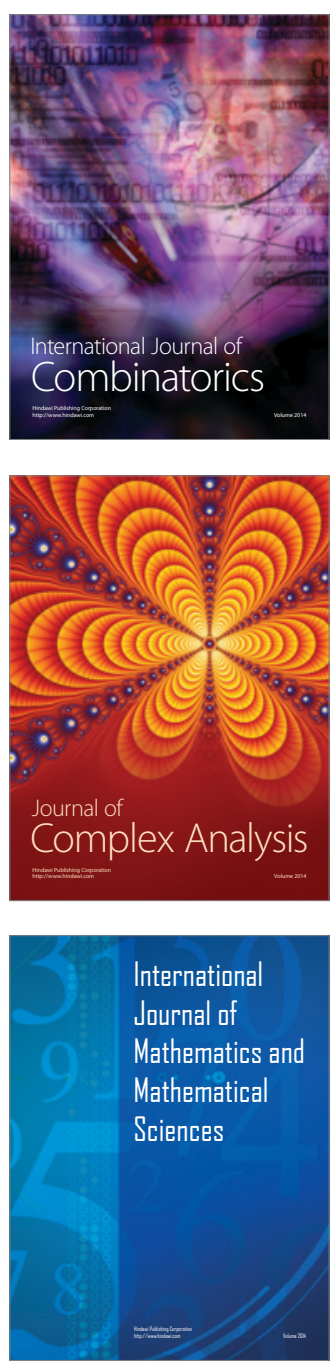
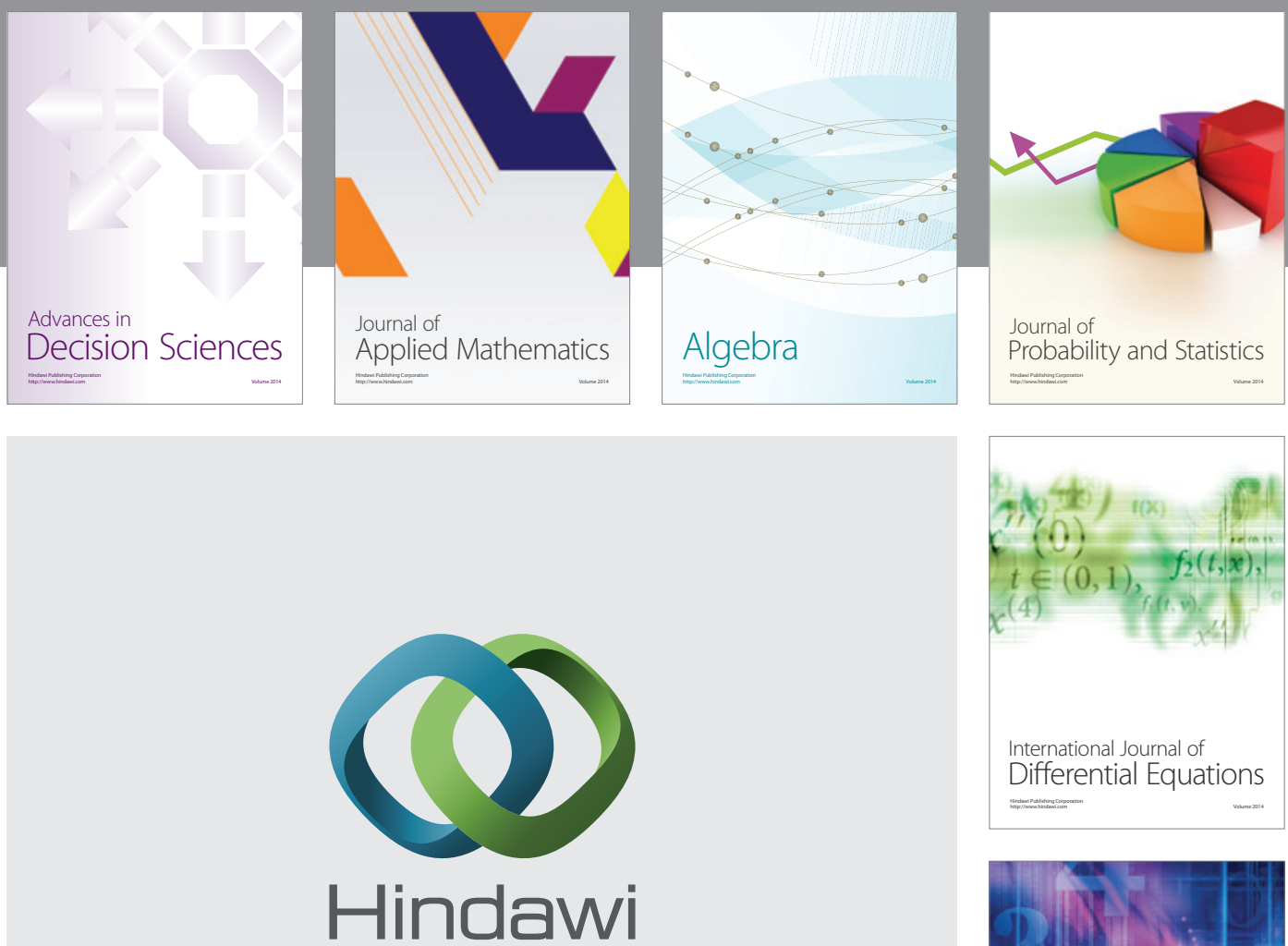

Submit your manuscripts at http://www.hindawi.com
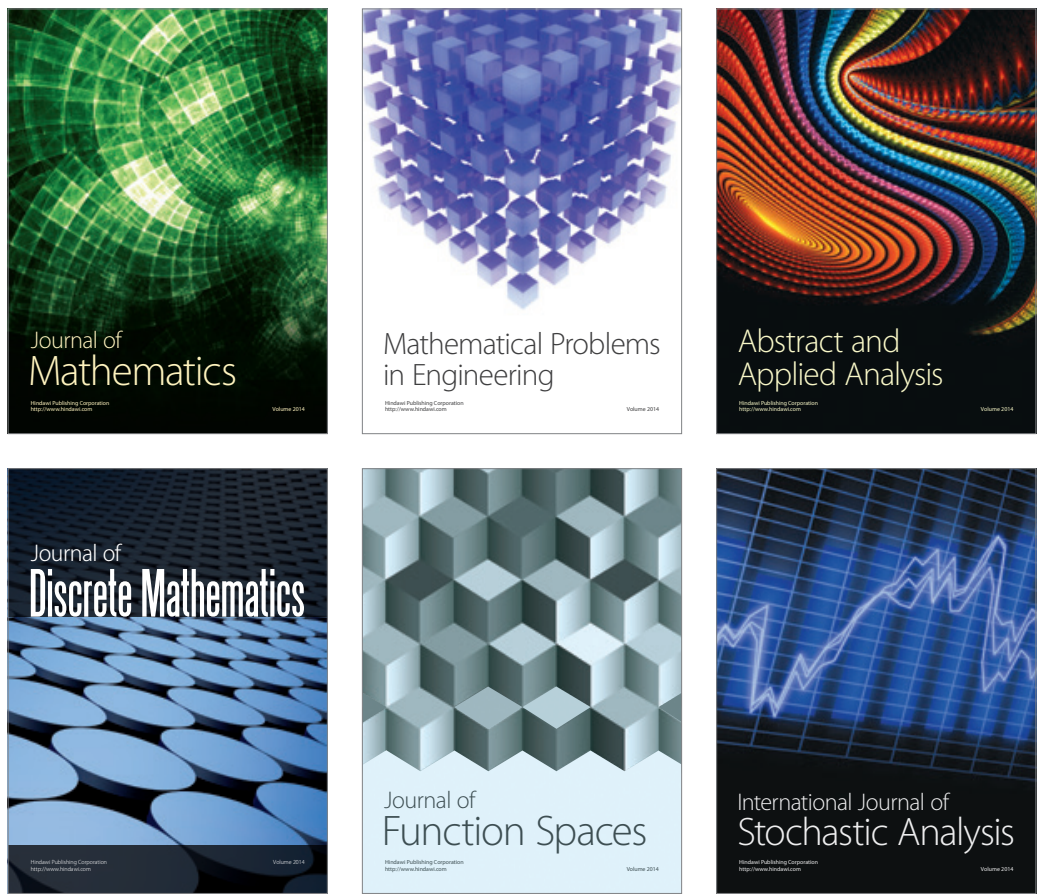

Journal of

Function Spaces

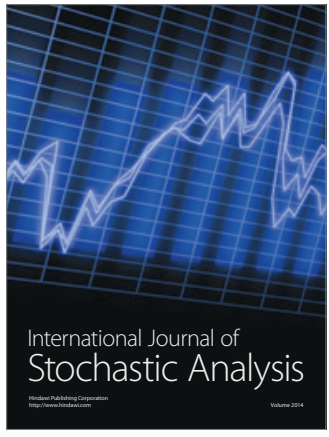

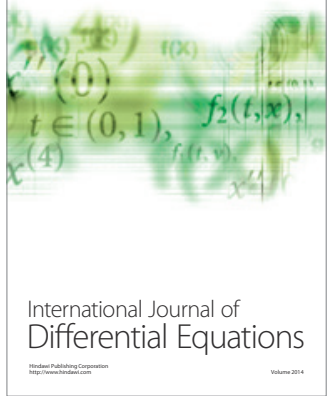
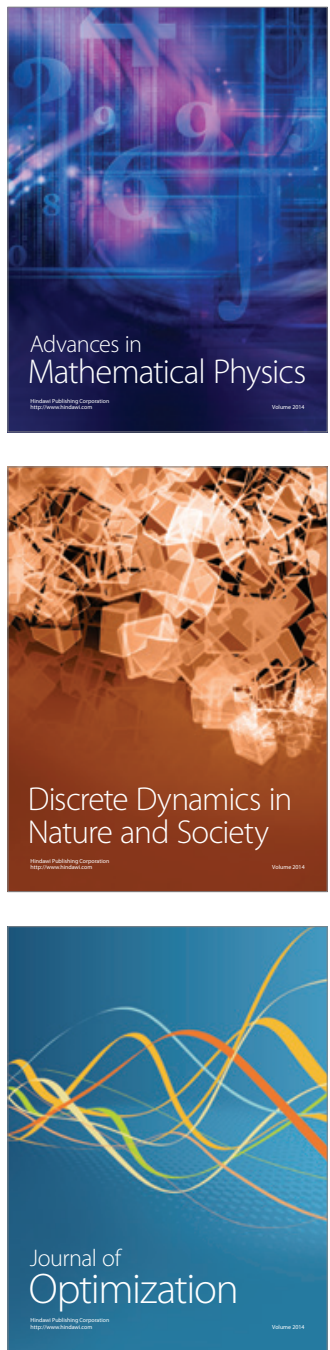